\title{
Taxonomic review of the Sphecapatodes ornata group (Diptera: Sarcophagidae: Miltogramminae), with description of one new species
}

\author{
Ming Zhang ${ }^{1 \dagger}$, Wen-Wen Chu ${ }^{2 \dagger}$, Thomas Pape ${ }^{3}$ and Dong Zhang ${ }^{1 *}$
}

\begin{abstract}
Background: The genus Sphecapatodes Villeneuve is recorded from China for the first time.

Results: The Sphecapatodes ornata group is defined and reviewed, with one new species, Sphecapatodes xuei sp. nov., described, photographed, and illustrated. The current concept of S. ornata Villeneuve, 1912 is shown to be based at least partly on a misidentification. A key to males of the S. ornata group is given. Wing interference patterns (WIPs) are presented for species-level discrimination of miltogrammine sarcophagids for the first time.

Conclusions: The genus Sphecapatodes Villeneuve, 1912 is represented in China by two species, S. kaszabi Rohdendorf and Verves, 1980 and S. xuei sp. nov. WIPs can be used for species-level discrimination of miltogrammine sarcophagids.
\end{abstract}

Keywords: Miltogramminae; Sphecapatodes; New species; Wing interference patterns; Taxonomy; China

\section{Background}

The flesh fly subfamily Miltogramminae makes up the sister group of all remaining sarcophagids (Pape 1996; Kutty et al. 2010). Miltogrammines are generally considered to be kleptoparasites of solitary bees and wasps, utilizing the stored food (insects, spiders, pollen) that these hymenopterans provide for their own progeny (Pape 1996, 1998; Povolný and Verves 1997; Szpila and Pape 2008), but recent evidence indicates that several species exploit buried carrion, which makes them of importance as forensic indicators in arid areas (Szpila et al. 2010; unpublished). Pape and Kurahashi (1995) mentioned that very few records of Miltogramminae have been published from China probably due to insufficient collecting. The arid climate and geomorphological configuration of northwestern China would seem to provide many different environments suitable to host a large diversity of Miltogramminae. With the aim of discovering more species of this group, we have been engaged in faunal studies in northwestern China, mainly focused on

\footnotetext{
*Correspondence: ernest8445@163.com

${ }^{\dagger}$ Equal contributors

'College of Nature Conservation, Beijing Forestry University, Beijing 100083, China

Full list of author information is available at the end of the article
}

the arid parts, since 2009. While sorting and identifying miltogrammine specimens from the Xinjiang Uygur Autonomous Region, the first author found specimens of the genus Sphecapatodes Villeneuve, which has previously not been recorded from China. Two species of Sphecapatodes were identified, one of which is considered to be new to science.

The genus Sphecapatodes was established by Villeneuve (1912), with S. ornata Villeneuve, 1912 as the type species fixed by monotypy. Séguy (1941) published the North African Sphecapatodes maroccanus as the second species in this genus; however, this species was revised and transferred to Miltogramma Meigen by Pape and Szpila (2012). Rohdendorf and Verves (1980) described Sphecapatodes kaszabi from Mongolia. Pape $(1996,1998)$ treated the genus Turkmenisca Rohdendorf as a junior synonym of Sphecapatodes, thus including additional three species, i.e., S. inornata (Rohdendorf 1975), S. fursovi (Rohdendorf 1975), and S. richterae (Verves 1980). In the present study, we describe one new species, bringing the total of known species of Sphecapatodes to six, three of which belong to the S. ornata group as defined below. The genus Sphecapatodes has its center of diversity in Central Asia, and so far only the type species $S$. ornata is 
known from the western Palaearctic, this species being described from Tunisia in North Africa.

The primary aims of this paper are to (i) define and review the S. ornata group; (ii) describe, photograph, and illustrate the new species $S$. xuei sp. nov.; (iii) provide a key to the known species of the $S$. ornata group; and finally (iv) use wing interference patterns (WIPs) for the separation of species for the first time in the subfamily Miltogramminae.

\section{Methods}

Photographs were taken with a Canon 500D camera (Canon, Inc., Tokyo, Japan) mounted on an Olympus SZX16 stereomicroscope (Olympus Corp., Tokyo, Japan). The images processing software used were Helicon Focus 3.2 (Helicon Soft Ltd, Kharkov, Ukraine) and Adobe Photoshop CS3 (Adobe Systems, Inc., San Jose, CA, USA). Methods for viewing and documenting interference color patterns in thin membranous wings are explained in Shevtsova et al. (2011) and Shevtsova and Hansson (2011). WIP images were produced using a 70-mm-diameter light-emitting diode (LED) ring light fitted on a stereomicroscope with a working distance of $60 \mathrm{~mm}$, giving an angle between lines of observation and incident light beams of about $40^{\circ}$. Terminology of adult morphology follows McAlpine (1981), and the male terminalia follows Sinclair (2000).

\section{Results}

\section{Genus Sphecapatodes Villeneuve}

\section{Nomenclature}

According to Article 30.1.4.4 of the International Code of Zoological Nomenclature (ICZN 1999), 'A compound genusgroup name ending in the suffixes -ites, -oides, -ides, -odes, or -istes is to be treated as masculine unless its author, when establishing the name, stated that it had another gender or treated it as such by combining it with an adjectival species-group name in another gender form.' Therefore, when Villeneuve (1912) described Sphecapatodes and combined this new monotypic genus with the speciesgroup epithet ornata, which is an adjectival word in the feminine form (of the Latin ornatus, $-a$, $-u m$, meaning decorated), he also fixed the gender of Sphecapatodes as feminine. Rohdendorf (1975), Verves (1986), and Pape (1996) are thus in error when treating the genus as masculine.

\section{Taxonomy}

Sphecapatodes Villeneuve, 1912: 508. Type species: S. ornata Villeneuve, 1912, by monotypy.

Turkmenisca Rohdendorf, 1975: 220. Type species: T. inornata Rohdendorf, 1975, by original designation [synonymized by Pape (1996)].
For detailed diagnostic information on this genus, see Pape (1996, 1998) and Pape and Szpila (2012).

\section{Sphecapatodes ornata group}

Diagnosis. The S. ornata group is proposed here on the basis of the following morphological characteristics that differentiate the group from other members of the genus Sphecapatodes: the posterior part of frontal vitta (at vertex) which is three times as broad as its anterior part (just before lunule); frontal bristles that are fine and not crossed; first flagellomere is short, about $1.20 \times$ as long as the pedicel (Figures 1B, 2B, 3B, 4B, and $5 \mathrm{~B}$ ); and male fore tibia, slightly narrower in basal one third. The $S$. ornata group consists of all the known species of Sphecapatodes with the exclusion of the three originally described in Turkmenisca, but the latter may well be paraphyletic with regard to the former as they have not been shown to share any synapomorphies, hence, we maintain the synonymy established by Pape (1996).

Catalog of the known species of the $S$. ornata group

Sphecapatodes kaszabi Rohdendorf and Verves

(Figures 1, 2, 3, 6, 7A,B, and 8A,C)

Arabisca dimorpha Rohdendorf, 1937: 46. Nomen nudum.

Sphecapatodes ornatus sensu Rohdendorf (1975: 217) (misidentification).

S. kaszabi Rohdendorf and Verves, 1980: 512. Type locality: Mongolia, Bayan Khongor Aimak, Ekhin Gol 90 km N Caganbulak; Pape 1996: 144.

Redescription. (Based on the newly collected specimens from Xinjiang, photos of type specimens as well as the original description in Russian). Male. Body length 6.10 to $7.00 \mathrm{~mm}$.

Eyes, bare. Fronto-orbital, parafacial plates, and postocular strip black with silvery white pollinosity; parafacial plate with rows of fine setulae, denser at the lower part; fronto-orbital plate with one row of setulae. Frontal vitta black, with thick silvery white pollinosity, becoming narrow at the anterior (lower) part; frons at vertex 0.40 to $0.45 \times$ head width; frontal vitta with several fine setulae; frontal row of 8 to 12 bristles; outer vertical bristle differentiated from postocular bristles and about $0.60 \times$ as long as inner vertical bristle, proclinate orbital bristles four (two strong), reclinate orbital bristle one. Ocellar bristles directed laterally. Gena ground color brown, with sparse and short black setulae and silvery gray pollinosity, 0.18 to $0.20 \times$ eye height in lateral view. Antenna dark brown, first flagellomere $1.20 \times$ as long as pedicel; arista black brown, bare, or micro-pubescent, basal one fifth swollen; facial ridge with one or two fine setulae at the lower part. Palpus orange, slightly expanded at the apex.

Thorax, ground color black; scutum and scutellum with thick grayish white pollinosity. Chaetotaxy: acrostichals 


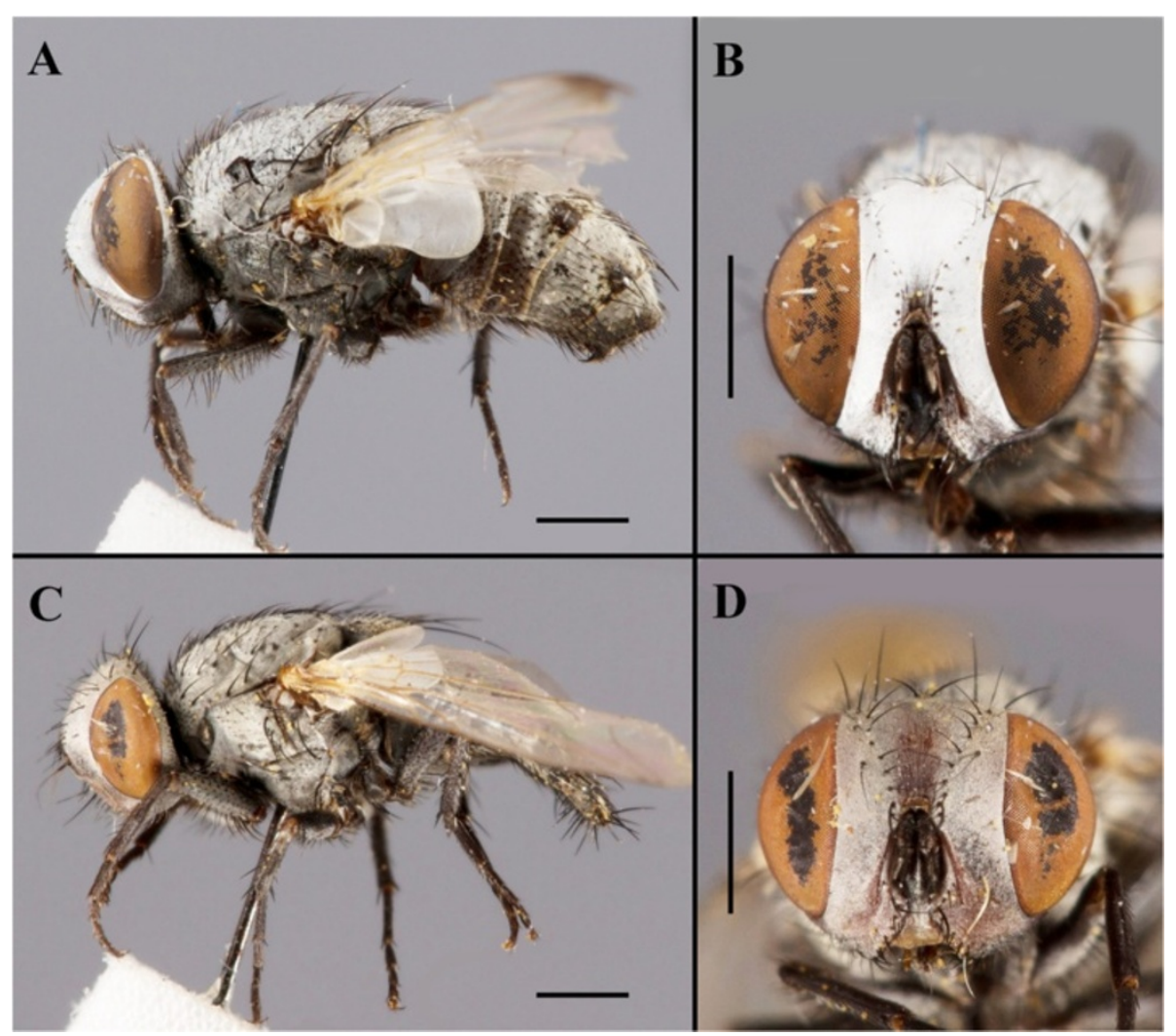

Figure 1 Sphecapatodes kaszabi Rohdendorf and Verves, 1980. Holotype $\delta$ and paratype $q$ from Mongolia. (A) Male habitus, left lateral view. (B) Male head, anterior view. (C) Female habitus, left lateral view. (D) Female head, anterior view. Scale bars, A to D = $1.00 \mathrm{~mm}$. (A to D reproduced through the courtesy of Mr. Zoltán Soltész).

$1(2)+1$, dorsocentrals $2+3$, intra-alars $1+2$, supra-alars 2 , postpronotals 2 , postalars 2 , notopleurals 2 , scutellum with 2 discal, and 3 pairs of marginal bristles. Pleuron with five to eight meropleurals, katepisternal bristles $1+1$, prosternum, metasternum, proepisternum, and postalar wall bare.

Wing hyaline, with one faint apical spot; subcostal sclerite and basicosta bare and yellow; tegula dark yellow, with black setulae; costal spine not differentiated; vein $R_{1}$ bare; several fine black setulae at node of $R_{4+5^{-}}$ $\mathrm{R}_{2}+3$; wing WIP (Figure 8A) with a clearly demarcated green band between an inner magenta band and an outer blue/magenta band, with a narrow yellow band along posterior wing margin, and with a green spot on the apical part (shown with a star in Figure 8A).

Legs dark, claws and pulvilli small; fore femur posteriorly with numerous long bristles, one ventral row of bristles, fore tibia slightly narrower in basal one third, with one median posteroventral, one subapical anterodorsal and one dorsal bristle, fore first tarsomere with dense setulae on the ventral surface at base and with sparse setulae on the anteroventral and posteroventral surfaces (Figure 7A,B); all tarsomeres of fore leg with one elongate, slender seta apico-distally; mid-femur with one row of anteroventral bristles, three anterodorsal, and two apical posterodorsal bristles, and mid-tibia with three anterodorsal, one submedian ventral, and one row of posterodorsal bristles; hind femur with dense setulae on anteroventral and ventral surface, one dorsal row of bristles, hind tibia with one anterodorsal row of bristles, and four ventral and four posterodorsal bristles.

Abdomen with dense gray pollinosity; tergites $1+2$ with 3 distinct black spots, the median one large, tergite 3 with a black median stripe, and with one pair of median marginal bristles; tergite 4 with inconspicuous median spot, the lateral spot small, and with a complete row of marginal bristles; sternites 1 and 2 with long and dense setulae. Terminalia: Cercus small and gently curved distally, surstylus with an oval rounded tip in lateral view, phallus parallel-sided distal to the dorsal plate and rounded apically (Figure 6).

Female. Body length 6.00 to $7.00 \mathrm{~mm}$. Differs from male in the following characters:

Head. Frons at vertex 0.46 to $0.49 \times$ as broad as head width. Thorax, scutum, and scutellum with gray pollinosity. Chaetotaxy: acrostichals $3(4)+1$. Wing without apical spot. Fore tibia not narrow in basal part, with one posterior and three weak anterodorsal bristles; mid-tibia 


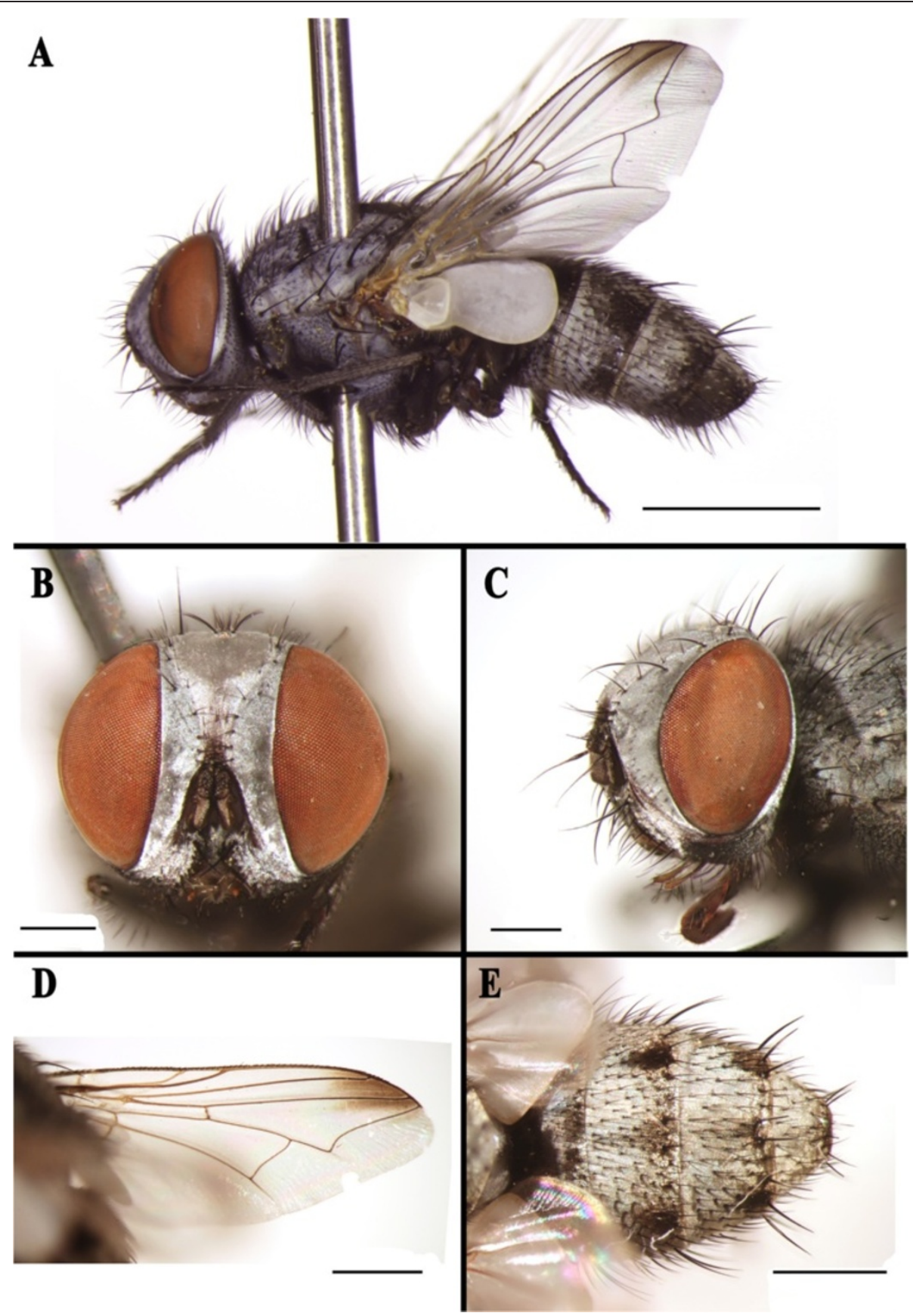

Figure 2 Sphecapatodes kaszabi Rohdendorf and Verves, 1980. Male, from Xinjiang, China. (A) Habitus, left lateral view. (B) Head, anterior view. (C) Head, left anterolateral view. (D) Right wing, dorsal view. (E) Abdomen, dorsal view. Scale bars, $\mathbf{A}=2.00 \mathrm{~mm} ; \mathbf{B}$ and $\mathbf{C}=0.50 \mathrm{~mm}$; D and $\mathbf{E}=1.00 \mathrm{~mm}$.

with one ventral and one posterior bristle; hind tibia with two ventral and three posterodorsal bristles. Abdominal tergites, 3 to 5 with complete marginal rows, tergite 3 with an inconspicuous black median stripe; tergites 4 and 5 without median black spot; sternites 1 and 2 with sparse setulae (two large).

Specimens examined. Mongolia: holotype $\hat{\delta}$ and paratype + (from photographs), Bayan Khongor Aimak, Ekhin
Gol 90 km N Caganbulak, altitude 950 m, 1967-VI-27-29, all collected by Z. Kaszab (specimens deposited in HNHM); Kazakhstan: $2 \hat{\jmath}, 1$, Almaty Province, Balkhash District, River Ili, $50 \mathrm{~km}$ downstream from Bakanas, 1953-V-3-17, all collected by G. Viktorov (specimens deposited in ZMUM); Turkmenistan: 1 त, Zakaspijskaja (=Transcaspian) Oblast, Türkmenbaşy (=Turkmenbashi or Krasnovodsk), 'V.900' (probably 


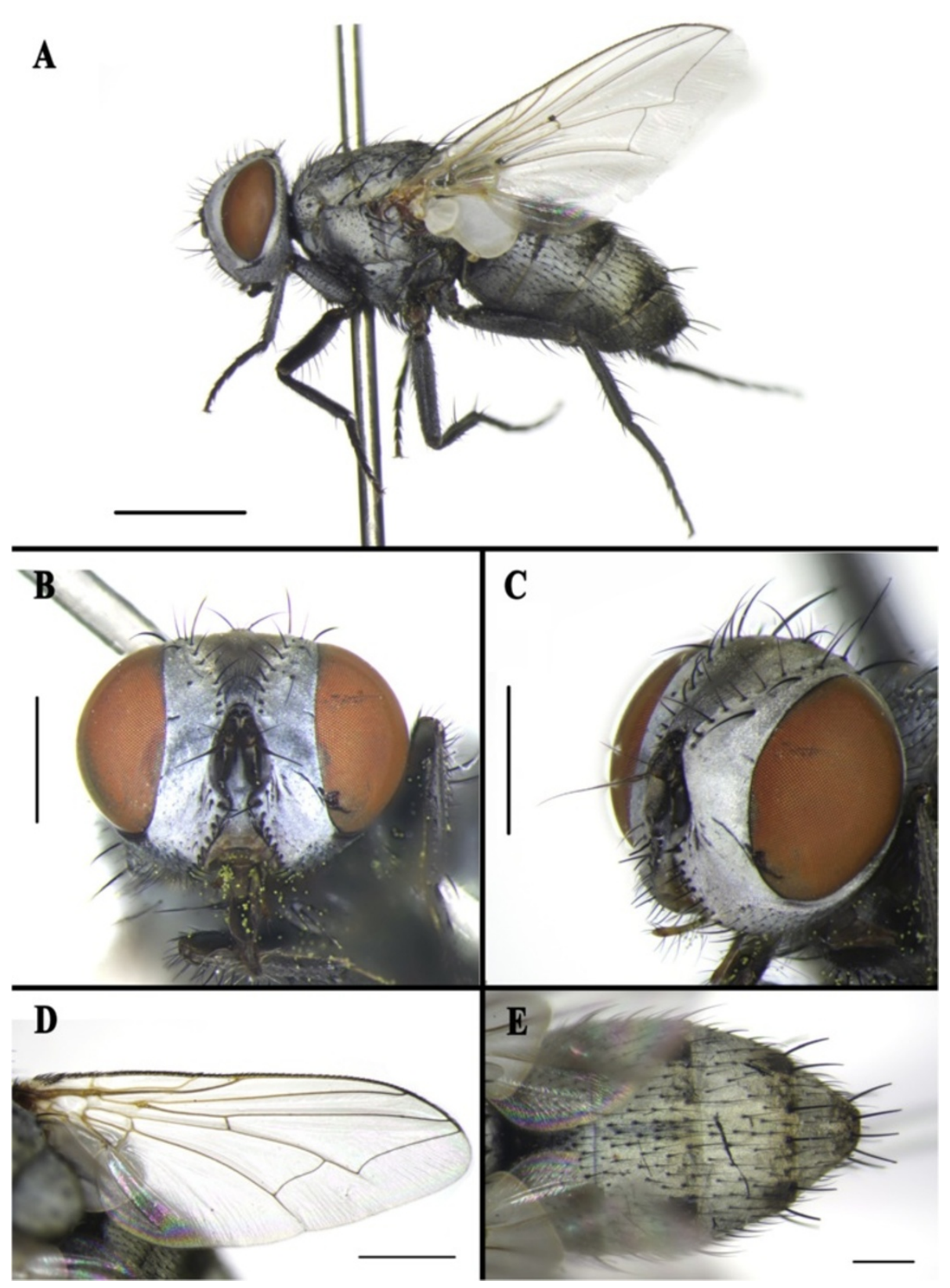

Figure 3 Sphecapatodes kaszabi Rohdendorf and Verves, 1980. Female, from Xinjiang, China. (A) Habitus, left lateral view. (B) Head, anterior view. (C) Head, left anterolateral view. (D) Right wing, dorsal view. (E) Abdomen, dorsal view. Scale bars, $\mathbf{A}=2.00 \mathrm{~mm} ; \mathbf{B}$ to $\mathbf{D}=1.00 \mathrm{~mm} ; \mathbf{E}=0.50 \mathrm{~mm}$.

1900-V), collected by 'Ancherv' (=abbreviated collector's name) (specimen deposited in ZMUM); China: 4 ถิ $\hat{0}$, 5 우오, Xinjiang, Altay, Kalamaili $\left(44^{\circ} 51^{\prime} 36^{\prime \prime} \mathrm{N} 89^{\circ} 5^{\prime}\right.$

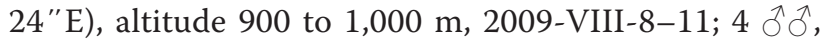
Xinjiang, Altay, Kalamaili, 2010-V-26; all collected by D. Zhang; 52 ๙ิ $\widehat{~}, 12$ 우, 2014-V-15-VI-16, Xinjiang, Altay, Kalamaili, all collected by M. Zhang (specimens deposited in MBFU).

Biology. The female specimen documented for the present paper (Figure 3B,C) has numerous pollen grains sticking to the setulae of the proboscis and on the fore legs, strongly indicating that the adults of this species have a habit of visiting flowers.

Distribution. China [Xinjiang (first record)], Kazakhstan (first record), Turkmenistan (first record), Mongolia.

Remarks. As mentioned under S. ornata (below), we consider Rohdendorf's (1975) concept of 'Sphecapatodes ornatus' to be a misidentification, as at least the specimens from ZMUM which Rohdendorf named 'S. ornatus' are conspecific with S. kaszabi. Comparing the original 


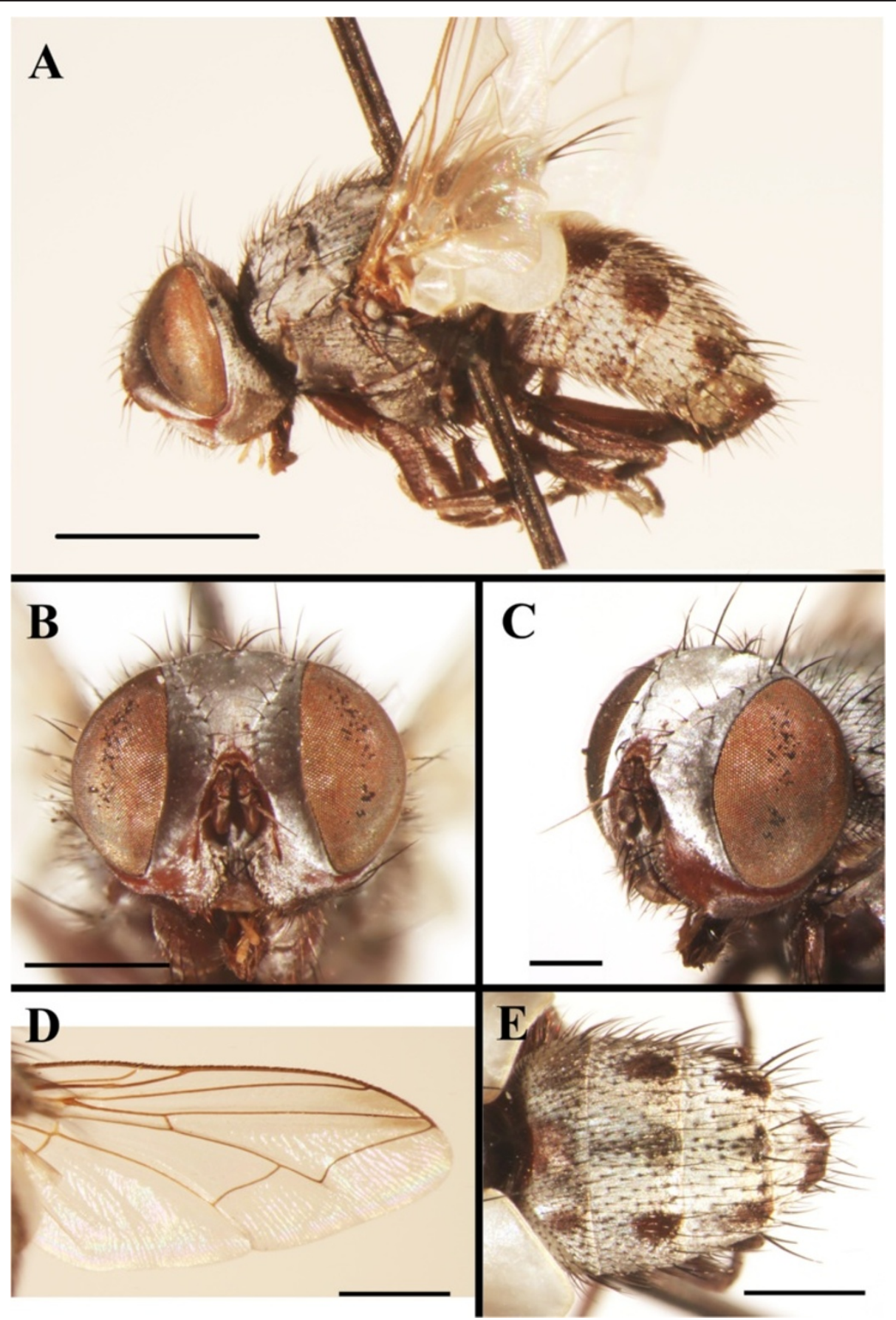

Figure 4 Sphecapatodes ornata Villeneuve, 1912. Male, syntype from Tunisia. (A) Habitus, left lateral view. (B) Head, anterior view. (C) Head, left anterolateral view. (D) Right wing, dorsal view. (E) Abdomen, dorsal view. Scale bars, $\mathbf{A}=2.00 \mathrm{~mm} ; \mathbf{B}=1.00 \mathrm{~mm} ; \mathbf{C}=0.50 \mathrm{~mm} ; \mathbf{D}$ and $\mathbf{E}=1.00 \mathrm{~mm}$.

description of $S$. kaszabi as well as the photographs of the holotype with newly collected specimens, we were unable to find differences justifying more than a single species, which has led us to consider all the examined specimens to be conspecific.

Sphecapatodes ornata Villeneuve

(Figures 4, 7C, and 8B)
S. ornata Villeneuve, 1912: 508. Type locality: Tunisia; Pape 1996: 144; Pape 1998: 666.

Redescription. Male. Body length 4.80 to $7.60 \mathrm{~mm}$.

Eyes, bare. Fronto-orbital and postocular strip black with silvery white pollinosity; parafacial plate yellow with two rows of fine setulae. Frontal vitta black, with thick silvery white pollinosity, becoming narrow at the 


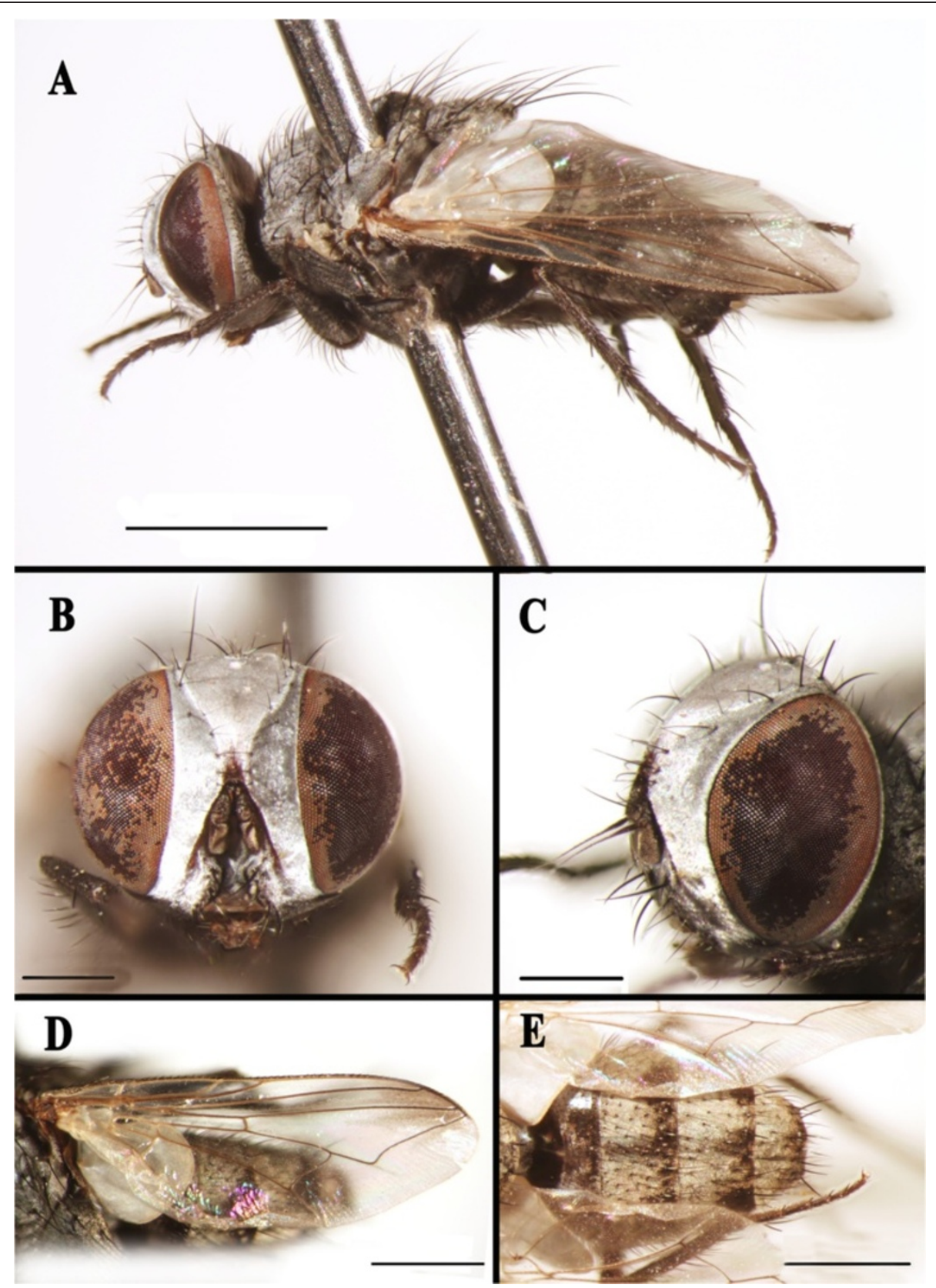

Figure 5 Sphecapatodes xuei sp. nov. Male, holotype from Xinjiang, China. (A) Habitus, left lateral view. (B) Head, anterior view. (C) Head, left anterolateral view. (D) Right wing, dorsal view. (E) Abdomen, dorsal view. Scale bars, $\mathbf{A}=2.00 \mathrm{~mm} ; \mathbf{B}$ and $\mathbf{C}=0.50 \mathrm{~mm} ; \mathbf{D}$ and $\mathbf{E}=1.00 \mathrm{~mm}$.

anterior part; frons at vertex $0.45 \times$ head width; frontal row of 8 to 12 bristles; outer vertical bristle differentiated from postocular bristles and about $0.75 \times$ as long as inner vertical bristle, and proclinate orbital bristles four (two strong) and reclinate orbital bristle one. Ocellar bristles directed laterally. Gena ground color dark red, with sparse and short black setulae and silvery gray pollinosity, $0.15 \times$ eye height in lateral view. Antenna grayish brown, first flagellomere $1.20 \times$ as long as pedicel; arista black brown and micro-pubescent, basal one fifth swollen; facial ridge with three fine setulae at the lower part. Palpus orange, slightly expanded at the apex.

Thorax, ground color black; scutum with gray pollinosity. Chaetotaxy: acrostichals $3(4)+1$, dorsocentrals $3+4$, intra-alars $0+1$, supra-alars 2 , postpronotals 2 , postalars 2 , notopleurals 2 , scutellum with 1 discal and 3 marginal bristles. Pleuron with 6 or 7 meropleurals, katepisternal bristles $1+1$, prosternum, metasternum, proepisternum, and postalar wall bare. 


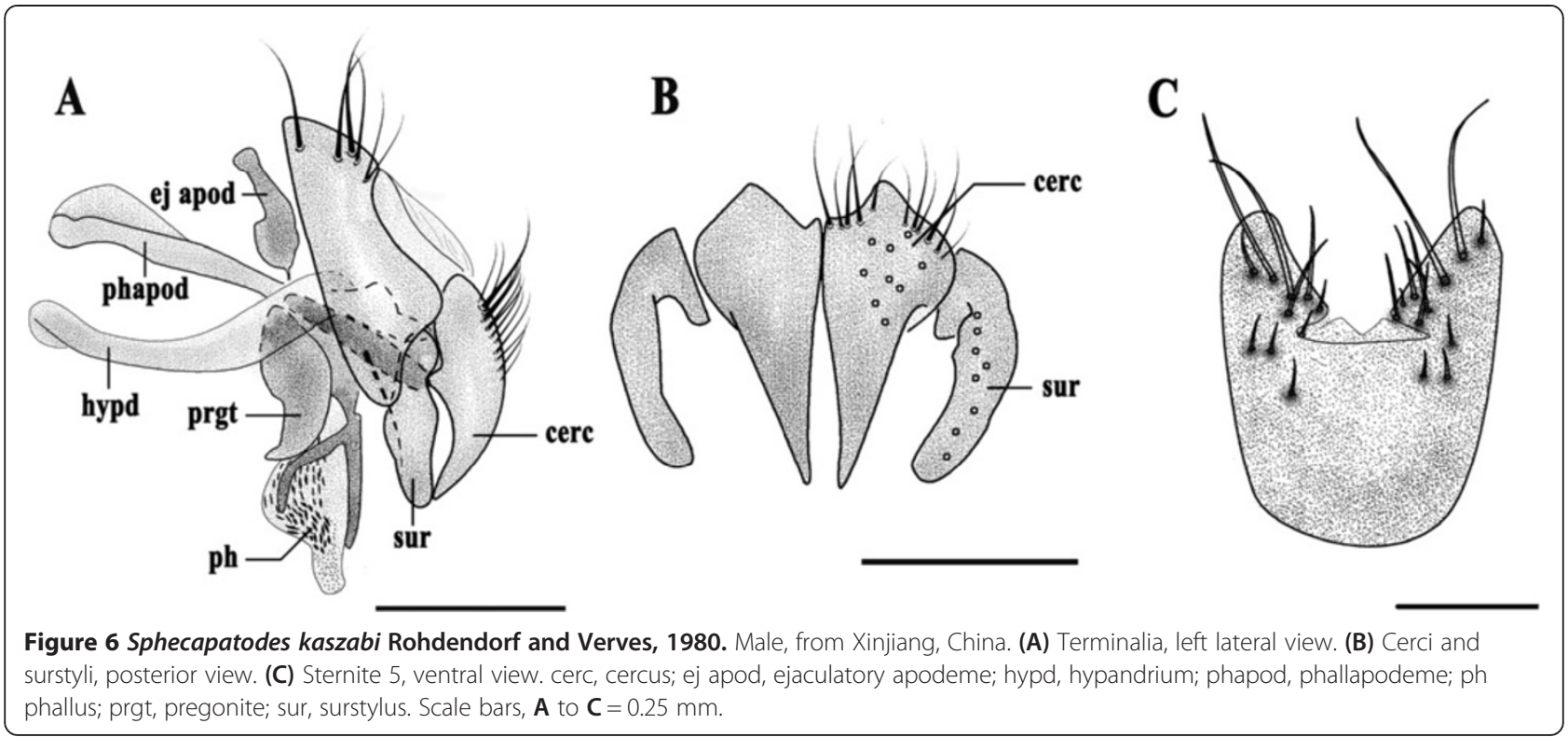

Wing hyaline, with apical spot faint and diffuse; subcostal sclerite and basicosta bare and yellow; tegula dark yellow, with black setulae; costal spine not differentiated; vein $R_{1}$ bare; one ventral and two dorsal black setulae at node of $R_{4}+5-R_{2}+3$; wing WIPs (Figure $8 B$ ) with a broad yellow band along posterior wing margin and with a magenta spot on the apical part (shown with star).

Legs dark, claw and pulvillus small; fore femur posteriorly with numerous long setulae, one ventral row of bristles, fore tibia slightly narrower in basal one third, with one median posteroventral bristle, one subapical dorsal bristle, five anterodorsal bristles, fore first tarsomere with dense setulae on the anteroventral surface (Figure 7C); mid-femur with one row of ventral bristles, one apical posterodorsal and one apical posterodorsal bristles, mid-tibia with two anterodorsal, one submedian anteroventral, and two posterior and two posterodorsal bristles; hind femur with one dorsal row of bristles, hind tibia with one anterodorsal row of bristles, and two anteroventral and two posterodorsal bristles.

Abdomen tergites $1+2$, tergite 3 and 4 with complete rows of marginal bristles, tergites $1+2$ with 3 distinct
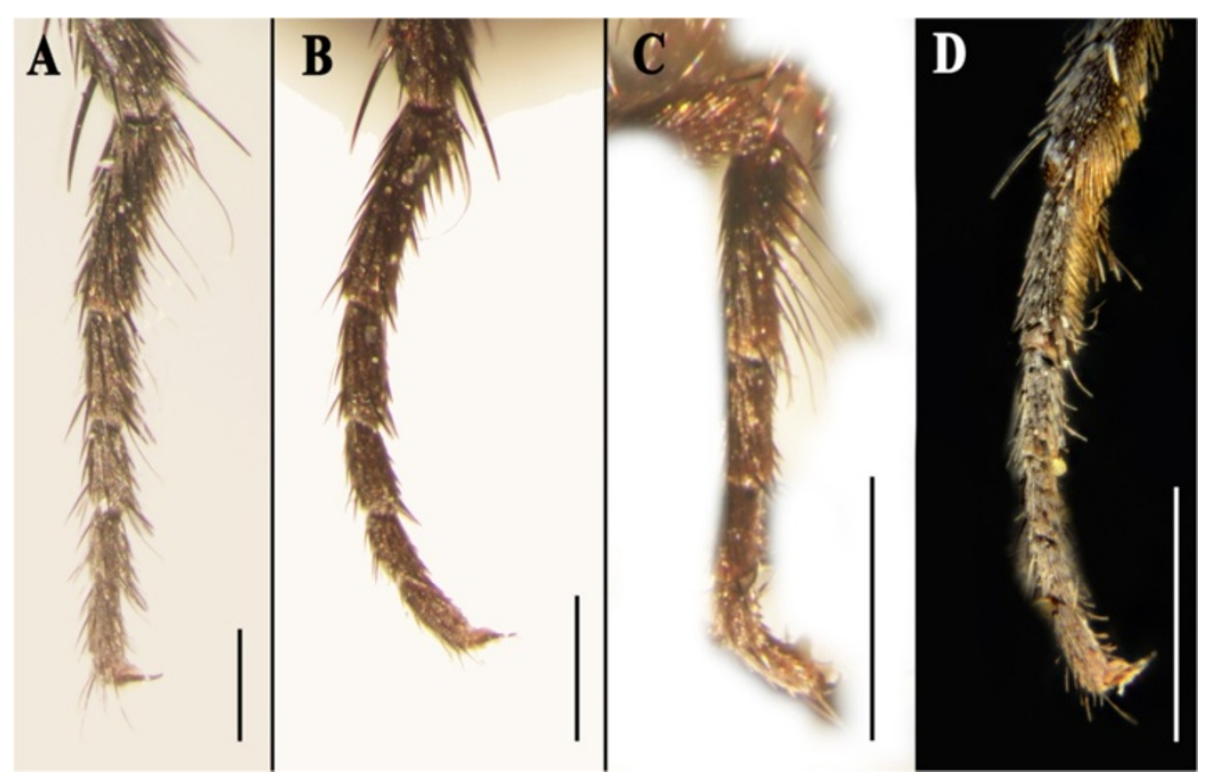

Figure 7 Photographs of the male fore tarsi of Sphecapatodes spp., posterior view. (A) S. kaszabi, specimen from Xinjiang, China. (B) S. kaszabi, holotype from Mongolia. (C) S. ornata, syntype from Tunisia. (D) S. xuei sp. nov., holotype from Xinjiang, China. Scale bars, A and $\mathbf{D}=0.25 \mathrm{~mm} ; \mathbf{C}=0.25 \mathrm{~mm}$. Arrows in (A) and (B) show variation in the diagnostic character of the fore tarsus of S. kaszabi (B reproduced through the courtesy of Mr. Zoltán Soltész). 

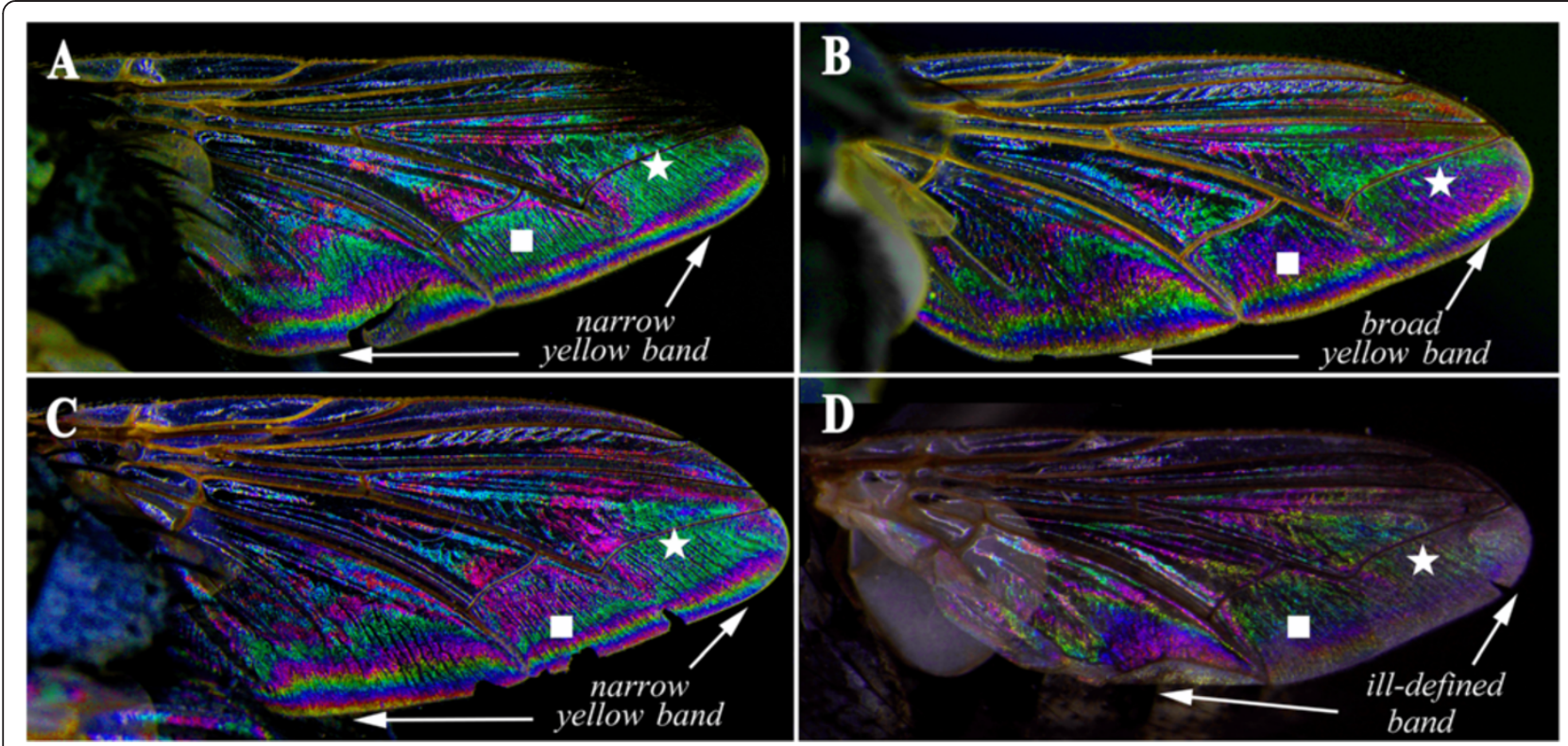

Figure 8 WIPs of the right wing of Sphecapatodes spp., dorsal view. (A) S. kaszabi, male specimen from Xinjiang, China. (B) S. ornata, male, syntype from Tunisia. (C) S. kaszabi, female, specimen from Xinjiang, China. (D) S. xuei sp. nov., male, holotype from Xinjiang, China. Squares and stars show the most obvious differences between the three species of the Sphecapatodes ornata group.

black spots, the median one large, median spots of tergites 3 and 4 smaller than the lateral ones.

Female. Unknown.

Material examined. Syntypes, $2 \AA \hat{\partial}$, Tunisia (no further locality data) (specimens deposited in ZMUC).

Distribution. Tunisia.

Remarks. S. ornata was recorded from Armenia, Azerbajdzhan, Egypt, Tajikistan, Turkmenistan, and Uzbekistan by Rohdendorf (1975) and Pape (1996), but these records appear to be based on misidentifications. It is noteworthy that Rohdendorf (1975) did not have access to Villeneuve's original material of $S$. ornata, and his redescription of this species deviates strikingly from the syntypes examined for the present paper in mentioning three or four long, fine setulae along the posterior surface (der Außenseite) of the male fore tarsus. The morphological similarities between the species Rohdendorf (1975) redescribed as S. ornatus and the species $S$. kaszabi appear to be consistent by careful comparison of material identified by Rohdendorf, that is to say, the S. ornatus Rohdendorf (1975) redescribed is in fact $S$. kaszabi (see remarks under this species).

Sphecapatodes xuei sp. nov.

(Figures 5, 9, 7D, and 8D)

Description. Male. Body length $6.00 \mathrm{~mm}$.

Eyes, bare. Fronto-orbital, parafacial plates, and postocular strip black with silvery white pollinosity; parafacial plate with one row of fine setulae; fronto-orbital plate with one or two fine setulae. Frontal vitta black, with
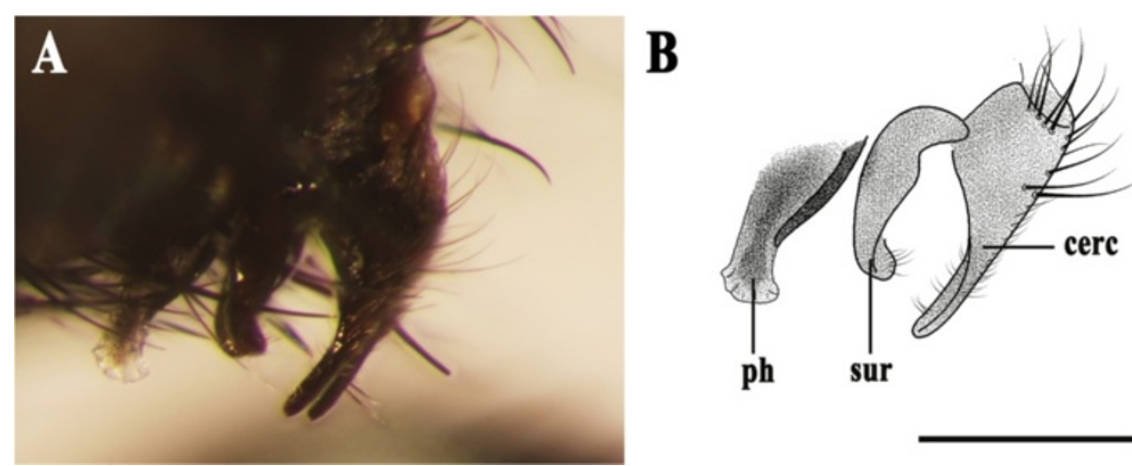

Figure 9 Sphecapatodes xuei sp. nov. Male, holotype from Xinjiang, China. (A) Tip of male abdomen, left lateral view, showing phallus, surstylus and cercus (holotype). (B) Phallus, surstylus and cercus, left lateral view (holotype). Scale bar $=0.25 \mathrm{~mm}$. cerc, cercus; ph, phallus; sur, surstylus. 
thick silvery white pollinosity and several fine setulae, becoming narrow at the anterior (lower) part; frons at vertex $0.43 \times$ head width; frontal row of six or seven bristles; outer vertical bristle differentiated from postocular bristles and about $0.60 \times$ as long as inner vertical bristle, proclinate orbital bristles two, reclinate orbital bristle one. Ocellar bristles directed laterally. Gena ground color brown, with sparse and short black bristles and silvery gray pollinosity, about $0.15 \times$ eye height in lateral view. Antenna dark brown, first flagellomere $1.10 \times$ as long as pedicel; arista bare and black brown, swollen in basal one fifth; facial ridge with one or two fine setulae at the lower part. Palpus orange, slightly expanded at the apex.

Thorax, ground color black; scutum and scutellum with thick grayish white pollinosity. Chaetotaxy: acrostichals $1+2$, dorsocentrals $2+3$, intra-alars $1+2$, supra-alars 1 , postpronotals 2 , postalars 2 , notopleurals 2 , scutellum with one discal and four marginal bristles. Pleuron with six or seven meropleurals, katepisternal bristles $1+1$, prosternum, metasternum, proepisternum, and postalar wall bare.

Wing hyaline, with one faint apical spot; subcostal sclerite and basicosta bare and yellow; tegula dark yellow, with black setulae; costal spine not differentiated; vein $R_{1}$ bare; several fine black setulae on dorsal surface at node of $R_{4+5}$ to $R_{2}+3$; wing WIP (Figure 8D) with a broad and somewhat ill-defined magenta band (tinged with yellow) near the posterior margin extending from wing tip to $\mathrm{CuA} 1$ and two larger and almost triangular magenta areas proximal to the middle of cell $\mathrm{R}_{4+5}$ and distally in the cubital cell.

Legs dark, claws and pulvilli small; fore femur posteriorly with numerous long setulae, one ventral row of bristles, fore tibia slightly narrower in the basal one third, with one median posteroventral, one subapical dorsal, and three anterodorsal bristles, fore first tarsomere with the usual dense setulae on the ventral surface at the base (Figure 7D) and with sparse setulae on the anteroventral and posteroventral surfaces; midfemur with one row of anteroventral bristles and two apical posterodorsal bristles, mid-tibia with one anterodorsal, one ventral, one posterior and one posterodorsal bristles; hind femur with one row of ventral bristles, hind tibia with one row of anterodorsal bristles (two bristles larger than the others) and one posterodorsal row of bristles (three bristles larger than the others) and three anteroventral bristles.

Abdomen with dense gray pollinosity; tergites $1+2$ with 3 black spots, almost fused together, spots on tergites 3 and 4 fused together at the posterior margin; tergites 3 to 5 with complete marginal bristles; sternites 1 and 2 with long and dense setulae. Terminalia: Cercus small and gently curved distally, surstylus with apex bent posteriorly, distal part of phallus slightly broadened (Figure 9).
Female. Unknown.

Remarks. The new species is very similar to $S$. kaszabi but can be separated from this species by the characters given in the key.

Material examined. Holotype $\delta$, China: Xinjiang, Altay, Kalamaili ( $\left.44^{\circ} 51^{\prime} 36^{\prime \prime} \mathrm{N} 89^{\circ} 5^{\prime} 24^{\prime \prime} \mathrm{E}\right)$, altitude 900 to 1,000 m, 2009-VIII-17, collected by D. Zhang; paratype, 1 ô, Xinjiang, Altay, Kalamaili, 2014-V-19, collected by M. Zhang (specimens deposited in MBFU).

Etymology. The new species is named after Prof. Wangqi Xue in honor of his outstanding work on Chinese Diptera.

Biology. Unknown.

Distribution. China (Xinjiang).

Key to known species of the S. ornata group (males only)

1. The width of the posterior (upper) part of frontal vitta about $1.50 \times$ as broad as its anterior (lower) part, frontal bristles long and crossed.................species formerly assigned to genus Turkmenisca; see Rohdendorf (1975) and Verves (1980)

- The width of the posterior part of frontal vitta about $3.0 \times$ as broad as its anterior part, frontal bristles fine and not crossed.

2. Proclinate orbital bristles 2 , gena ground color brown, presutural acrostichal bristles 1 or 2, dorsocentrals $2+3$, fore first tarsomere with sparse setulae on the anteroventral surface (Figure 7A,B) .............................. 3

- Proclinate orbital bristles four (two strong), gena ground color dark red, presutural acrostichal bristles absent, dorsocentrals $3+4$, fore first tarsomere with dense setulae on the anteroventral surface (Figure 7C) ........... S. ornata Villeneuve

3. Fore first tarsomere with one elongated ventral seta (Figure 7D), parafacial plate with about ten setulae (Figure 5C), the black spots on tergites 1 to 4 almost fused together (Figure 5E), wing interference pattern (Figure 8D) with only a single, rather ill-defined magenta band along the posterior margin, surstylus with tip curving posteriorly, phallus apically slightly broadened in lateral view (Figure 9).....

S. xuei sp. nov.

- Fore first tarsomere with four elongated ventral setulae (Figure 7A), parafacial plate with more than 20 setulae (Figure 4C), black spots on tergites 1 to 4 distinct (Figure 4E), WIP (Figure 8A) with a clearly demarcated green band between an inner magenta band and an outer blue/magenta band, surstylus with apex straight (not curving posteriorly), phallus apically narrower in lateral view (Figure 6A,B).................................. $S$. kaszabi Rohdendorf and Verves 


\section{Discussion}

Pape (1996) mentioned a number of character states, which together will both diagnose and define the genusgroup taxon Sphecapatodes. He also stated that 'The genera Sphecapatoclea and Sphecapatodes are probably sister groups, and [...] they could as well be lumped into one genus.' Judging from the diagnoses provided, this was based on the sharing of a dense silvery microtomentose male frontal vitta, laterally directed ocellar bristles, and subequal fringes of the metathoracic spiracles. This leaves only two autapomorphies for the genus Sphecapatodes, both confined to the male: (1) elongated setae ventrally on the fore first tarsomere (Figure 7) and (2) wing with apical wing spot (Figures 2D, 4D, and 5D). Pape (1996) noted that 'exceptions occur' with regard to the presence of the wing spot. However, the only species of Sphecapatodes without the male wing spot is S. inornata, and this seems to be partly due to a misrepresented or misprinted illustration (Rohdendorf 1975, figure 160a, b), as Rohdendorf's key explicitly states that the wing margin of the male is very slightly light brown in the area of the apical spot of S. fursovi [sehr schwach hell gebräunt an der Stelle des Apikalfleckes der vorigen Art (p. 220)]. With only the holotype of S. inornata known, more specimens are needed to fully evaluate the wing spot character for this species. As evident from the present paper, the male fore tarsal ornamentation in Sphecapatodes is rather diverse and most markedly developed on the first tarsomere, and the elongated setae may be either ventral or a combination of anteroventral + posteroventral, obscuring homology assessment.

WIPs were recently discovered by Shevtsova et al. (2011) as a potential new character system in the transparent cuticle of extremely thin membranous insect wings, especially small Hymenoptera and Diptera, and the method has proven useful for the separation of species (Buffington and Sandler 2011; Hansson 2011; Shevtsova and Hansson 2011; Shevtsova et al. 2011). There is a paucity of WIP data in the Cyclorrhapha, although WIPs represent an intriguing model of morphological diversity that might increase our understanding of evolutionary trends (Shevtsova et al. 2011).

Shevtsova and Hansson (2011) demonstrated the usefulness of WIPs to separate species in the genus Achrysocharoides Girault (Hymenoptera: Eulophidae), where several species also displayed sexual dimorphism in these patterns. Conversely, Hansson and Shevtsova (2012) demonstrated that species of the genus Omphale Haliday (Hymenoptera: Eulophidae) do not have sexually dimorphic WIPs. The current data are very preliminary due to limited material and in particular the lack of females, but as evident from Figure $8 \mathrm{~A}, \mathrm{C}$, there is no difference in the male and female WIPs of $S$. kaszabi, and all three species are easily identified from their WIP. This indicates that WIPs have the potential both of being a useful tool for correctly associating male and female specimens, which is a well-known problem in many sarcophagid groups (Hall et al. 2009; Zhang et al. 2013), and as an additional species-specific character complex. Obtaining WIP data in the form of digital photographs is remarkably simple (Buffington and Sandler 2011; Shevtsova et al. 2011) and WIPs might therefore be a new and rapid way to identify adult sarcophagids and associate males and females.

\section{Conclusions}

The $S$. ornata group is defined and reviewed, with one new species, S. xuei sp. nov., described, photographed, and illustrated. There is no difference in the male and female WIPs of S. kaszabi, and all three species are easily identified from their WIP. WIPs can be used for species-level identifications in this genus and should be investigated further as a potentially useful identification character for other sarcophagid groups.

\section{Abbreviations}

HNHM: Hungarian Natural History Museum, Budapest, Hungary;

MBFU: Museum of Beijing Forestry University, Beijing, China;

ZMUC: Zoological Museum, Natural History Museum of Denmark,

University of Copenhagen, Copenhagen, Denmark;

ZMUM: Zoological Museum, Moscow State University, Moscow, Russia.

\section{Competing interests}

The authors declare that they have no competing interests.

\section{Authors' contributions}

$M Z, W W C, T P$, and DZ wrote the manuscript. MZ and WWC produced the photographs. DZ participated in the field work during which new material was collected and directed the study. All authors read and approved the final manuscript.

\section{Acknowledgements}

We wish to extend our sincerest thanks to Drs. Jacek Szwedo and Adam Stroiński (Museum and Institute of Zoology, Polish Academy of Sciences, Warsaw, Poland) for their assistance with the WIP method in this study and to Mr. Zoltán Soltész (Hungarian Natural History Museum, Budapest, Hungary) for providing photographs of S. kaszabi. Dr. Andrey Ozerov (Zoological Museum, Moscow State University) kindly arranged for a loan of Sphecapatodes spp. This study was supported by the Beijing Higher Education Young Elite Teacher Project (No. YETP0771), Program for New Century Excellent Talents in University (No. NCET-12-0783), and National Science Foundation of China (No. 31201741).

\section{Author details}

${ }^{1}$ College of Nature Conservation, Beijing Forestry University, Beijing 100083, China. ${ }^{2}$ College of Humanities and Development Studies, China Agricultural University, Beijing 100083, China. ${ }^{3}$ Natural History Museum of Denmark, University of Copenhagen, Universitetsparken 15, Copenhagen 2100, Denmark.

Received: 23 April 2014 Accepted: 21 July 2014

Published: 1 August 2014

\section{References}

Buffington ML, Sandler RJ (2011) The occurrence and phylogenetic implications of wing interference patterns in Cynipoidea (Insecta: Hymenoptera). Invertebr Syst 25:586-597, http://dx.doi.org/10.1071/IS11038

Hall M, Adams Z, Wyatt NP, Testa JM, Edge W, Nikolausz M, Farkas R, Ready PD (2009) Morphological and mitochondrial DNA characters for identification and phylogenetic analysis of the myiasis-causing flesh fly Wohlfahrtia magnifica and its relatives, with a description of Wohlfahrtia monegrosensis sp. n. Wyatt \& Hall. Med Vet Entomol 23:59-71, doi:10.1111/j.1365-2915.2008.00779.x 
Hansson C (2011) Cornugon (Hymenoptera: Eulophidae: Entedoninae) a new genus from tropical America including ten new species. Zootaxa 2873:1-26

Hansson C, Shevtsova E (2012) Revision of the European species of Omphale Haliday (Hymenoptera, Chalcidoidea, Eulophidae). ZooKeys 232:1-157, doi:10.3897/zookeys.232.3625

ICZN (1999) International Code of Zoological Nomenclature. Fourth edition adopted by the International Union of Biological Sciences. International Trust for Zoological Nomenclature, London

Kutty SN, Pape T, Wiegmann BM, Meier R (2010) Molecular phylogeny of the Calyptratae (Diptera: Cyclorrhapha) with an emphasis on the superfamily Oestroidea and the position of Mystacinobiidae and McAlpine's fly. Syst Entomol 35:614-635, doi:10.1111/j.1365-3113.2010.00536.x

McAlpine JF (1981) Morphology and terminology - adults. In: McAlpine JF, Peterson BV, Shewell GE, Teskey HJ, Vockeroth JR, Wood DM (eds) Manual of Nearctic Diptera, vol 1. Research Branch, Agriculture Canada Monograph, 27, Ottawa, pp 9-63

Pape T (1996) Catalogue of the Sarcophagidae of the world (Insecta: Diptera). Mems Entomol Int 8:1-558

Pape T (1998) Sarcophagidae. In: Papp L, Darva B (eds) Contributions to a manual of Palaearctic Diptera. General and applied dipterology. vol 3. Science Herald, Budapest, pp 649-678

Pape T, Kurahashi H (1995) Taxonomy, systematics and distribution of Senotainia navigatrix (Meijere) (Diptera: Sarcophagidae, Miltogrammatinae). Jpn J Ent 63:485-492

Pape T, Szpila K (2012) Taxonomy and nomenclature of Eremasiomyia macularis and Miltogramma maroccana (Diptera: Sarcophagidae: Miltogramminae). Can Entomol 144:169-181, doi:10.4039/tce.2012.14

Povolný D, Verves YG (1997) The flesh flies of Central Europe (Insecta, Diptera, Sarcophagidae). Spixiana Suppl 24:1-260

Rohdendorf BB (1937) Fam. Sarcophagidae. (P. 1). Fauna USSR 19:1-501 (in Russian with German summary)

Rohdendorf BB (1975) 64h. Sarcophaginae. In: Lindner E (ed) Die Fliegen der palaearktischen Region. Band 11, Schweizerbart, Stuttgart, pp 177-232

Rohdendorf BB, Verves YG (1980) On the fauna of Sarcophagidae (Diptera) of the Mongolian People's Republic. III. Miltogrammatinae. Insects of Mongolia 7:445-518, in Russian with English subtitle

Séguy E (1941) Études sur les mouches parasites. 2. Calliphorides, calliphorines (suite), sarcophagines et rhinophorides de l'Europe occidentale et méridionale. Encycl Ent (Ser A) 21:1-436

Shevtsova E, Hansson C (2011) Species recognition through wing interference patterns (WIPs) in Achrysocharoides Girault (Hymenoptera, Eulophidae) including two new species. ZooKeys 154:9-30, doi:10.3897/ zookeys.154.2158

Shevtsova E, Hansson C, Janzen DH, Kjærandsen J (2011) Stable structural color patterns displayed on transparent insect wings. Proc Natl Acad Sci USA 108:668-673, doi:10.1073/pnas.1017393108

Sinclair BJ (2000) Morphology and terminology of Diptera male terminalia. In: Papp L, Darvas B (eds) Contributions to a manual of Palaearctic Diptera. General and applied dipterology. vol. 1. Science Herald, Budapest, pp 53-74

Szpila K, Pape T (2008) Morphological diversity of first instar larvae in Miltogramma subgenus Pediasiomyia (Diptera: Sarcophagidae, Miltogramminae). Zool Anz 247:259-273, http://dx.doi.org/10.1016/j. jcz.2008.03.001

Szpila K, Voss JG, Pape T (2010) A new dipteran forensic indicator in buried bodies. Med Vet Entomol 24:278-283, doi:10.1111/j.1365-2915.2010.00883.x

Verves YG (1980) New and little known flies of the subfamilies Miltogrammatinae and Macronichiinae (Diptera, Sarcophagidae) from the Asiatic part of the USSR. Ént Obozr 59:914-924 (in Russian with English summary)

Verves YG (1986) Family Sarcophagidae. In: Soós Á, Papp L (eds) Catalogue of Palaearctic Diptera Calliphoridae-Sarcophagidae, vol 12. Akadémiai Kiadó, Budapest, pp 1-265
Villeneuve J (1912) Diptères nouveaux du nord Africain. Bull Mus Natn Hist nat, Paris 8:415-417, 505-511

Zhang D, Zhang M, Pape T, Gu CW, Wu W (2013) Sarcophaga (Hoa) flexuosa Ho (Diptera: Sarcophagidae): association of sexes using morphological and molecular approaches, and a redefinition of Hoa Rohdendorf. Zootaxa 3670:71-79, http://dx.doi.org/10.11646/zootaxa.3670.1.6

doi:10.1186/s40555-014-0048-9

Cite this article as: Zhang et al:: Taxonomic review of the Sphecapatodes ornata group (Diptera: Sarcophagidae: Miltogramminae), with description of one new species. Zoological Studies 2014 53:48.

\section{Submit your manuscript to a SpringerOpen ${ }^{\circ}$ journal and benefit from:}

- Convenient online submission

- Rigorous peer review

- Immediate publication on acceptance

- Open access: articles freely available online

- High visibility within the field

- Retaining the copyright to your article

Submit your next manuscript at $>$ springeropen.com 